Reprod. Nutr. Dévelop., 1981, 21 (5A), 705-714.

\title{
Effets sur la sécrétion pancréatique exocrine du porc de l'administration intraveineuse prolongée de glucose : application à l'étude des mécanismes de l'adaptation au régime alimentaire
}

\author{
par C. SIMOES NUNES, T. CORRING
}

avec la collaboration technique de Anne-Marie GUEUGNEAU, Georgette FREDOU et P. VAISSADE

Laboratoire de Physiologie de la Nutrition, I.N.R.A. 78350 jouy en Josas, France.

Summary. Effects of prolonged intravenous glucose perfusion on pig pancreatic exocrine secretion.

The effects of intravenous glucose perfusion were studied in the pancreatic juice of fisiulated pigs. Perfusions were performed either discontinuously for $3 \mathrm{hrs}$ in the fasted or for $6 \mathrm{hrs}$ a day over 6 days in the fed animal. Pancreatic juice volume, total proteins and total activities of chymotrypsin, amylase and lipase were always significantly lowered by glucose given intravenously. Amylase seemed to be the most inhibited of the three enzymes studied. It appeared that prolonged intravenous glucose perfusion could not reproduce in the pig the pancreatic amylase adaptation seen after oral administration of glucose.

\section{Introduction.}

Si le phénomène de l'adaptation de la sécrétion pancréatique exocrine aux constituants du régime alimentaire est bien connu chez de nombreuses espèces (Grossman, Greengard et Ivy, 1943 ; Imondi et Bird, 1967 ; Behrman et Kare, 1969 ; Clary ef al., 1969 ; Corring, 1975) il n'en est pas de même en ce qui concerne la nature des mécanismes impliqués. Les travaux réalisés depuis près de 40 années ont fourni un certain nombre de données qui sont à l'origine de quelques hypothèses. Il semble que l'on peut aujourd'hui retenir l'idée du rôle joué par les produits d'hydrolyse digestive des constituants alimentaires de la ration (Ben Abdeljlil, Visani et Desnuelle, 1963 ; Robberecht et al., 1971 ; Felber, Zermatten et Dick, 1974 ; Johnson, Hurwitz et Kretchmer, 1977 ; Corring, 1977). Ainsi, on sait que l'activité de l'amylase pancréatique n'est pas modifiée lorsqu'on substitue dans le régime alimentaire l'amidon par du glucose (Ben Abdeljlil, Visani et Desnuelle, 1963 ; Robberecht et al., 1971) et ce dernier a été considéré comme le facteur de déclenchement de l'adaptation de l'enzyme glycolytique du pancréas exocrine aux glucides alimentaires. Des résultats obtenus au cours d'éfudes précédentes (Simoes Nunes et Corring, 1979, 1980), il ressort que le glucose stimule l'activité de l'amylase pancréatique par action au niveau de la muqueuse intestinale 
proximale. D'autre part, l'injection intraveineuse unique de glucose entraîne une diminution de l'activité enzymatique ainsi que du volume et des protéines tolales sécrétés, ce qui confirme les résultats obtenus chez le chien par Nakajima et Magee (1970) et par Saito ef al. (1978). Toutefois, Lavau, Bazin et Herzog (1974) et Adler et Kern (1975), ont observé que la perfusion intraveineuse d'une solution très concentrée de glucose pendant plusieurs jours provoquait une augmentation de l'activité spécifique de l'amylase dans le tissu pancréatique. Le glucose, en concentration égale ou supérieure à 30 p. 100 , administré de façon prolongée par voie intraveineuse chez le rat serait, au contraire d'une injection unique, capable de reproduire l'adaptation de l'amylase pancréatique aux glucides alimentaires. On peut cependant s'interroger sur la signification physiologique du résultat obtenu par ces auteurs étant donné la quantité très importante de glucose administré au rat. Dans la poursuite des études sur les mécanismes de cette adaplation, il apparaît primordial de s'assurer de la validité de l'hypothèse d'un effet spécifique sur la sécrétion pancréatique d'une perfusion intraveineuse prolongée d'une solution de glucose, dont la concentration permet de reproduire l'apport digestif du produit d'hydrolyse de l'amidon provenant d'un régime hyperglucidique. L'étude rapportée a été effectuée chez le porc dans ce but.

\section{Matériel et méthodes.}

\section{Animaux ef alimentation.}

Dix porcs castrés de race Large White, âgés de 90 à 120 jours, d'un poids vif moyen de $49,9 \pm 1,8 \mathrm{~kg}$ ont été utilisés. Ils ont été munis de fistules chroniques du conduit pancréatique et du duodénum (Corring, Aumaitre et Rérat, 1972) et de la veine jugulaire externe droite. Les animaux ont été logés en cages métalliques individuelles, placées dans une salle isolée et ont été nourris avec un régime alimentaire équilibré contenant 38 p. 100 d'amidon (Simoes Nunes et Corring, 1979 : régime C) en deux repas par jour de $800 \mathrm{~g}$ chacun $(9$ et $16 \mathrm{~h}$ ).

Les essais onł commencé après 8 jours de récupération post-opératoire.

\section{Schéma expérimental.}

Le principe de l'expérimentation a consisté à étudier les effets d'une perfusion intraveineuse prolongée de solutions iso et hypertonique de glucose sur la sécrétion pancréatique exocrine, d'une part chez l'animal à jeun ef d'autre part chez l'animal nourri. Les effets ont été comparés à ceux de perfusions d'une solution isotonique de $\mathrm{NaCl}$.

1) Perfusions intraveineuses chez l'animal à jeun. - Cette étude a été effectuée chez quatre porcs, après un jeûne de $18 \mathrm{~h}$, les animaux ayant libre accès à l'eau de boisson. Deux solutions de glucose ont été utilisées : 5 p. 100 (isotonique) et 15 p. 100 (hypertonique) dans le but de tester un effet éventuel de l'hypertonicité sur la sécrétion pancréatique. Les perfusions intraveineuses ont débuté toujours à la même heure de la journée $(9 \mathrm{~h})$ et ont été réalisées à un débit de $8,33 \mathrm{ml} / \mathrm{min}$ selon la séquence suivante : $\mathrm{NaCl} 0,9$ p. 100 (1 h), glucose 5 ou 15 p. 100 (1 h), $\mathrm{NaCl} 0,9$ p. 100 (1 h), glucose 5 ou 15 p. 100 (2 h) et $\mathrm{NaCl} \mathrm{0,9} \mathrm{p.} 100(1 \mathrm{~h})$. 
Chaque animal a subi deux perfusions espacées d'au moins 5 jours, l'une avec la solution de glucose à 5 p. 100 ef l'auire avec celle à 15 p. 100.

2) Perfusions intraveineuses chez l'animal nourri. - Les essais ont été effectués chez les six porcs restants, consommant les deux repas par jour. Dans le but de tenter de reproduire l'apporí de glucose provoqué par l'ingestion d'un régime riche en amidon, $300 \mathrm{~g}$ de glucose ont été quotidiennement perfusés selon le schéma ci-après. Afin d'éviter la perfusion d'un volume trop important, le glucose a été administré en solution à 10 p. 100. Les perfusions intraveineuses ont été réalisées sur des durées expérimentales de plusieurs jours à raison de $6 \mathrm{~h}$ par jour et à un débit de $8,33 \mathrm{ml} / \mathrm{min}$. Elles ont débuté exactement avec la prise du repas de $9 \mathrm{~h}$ et ont été effectuées selon la séquence suivante : $\mathrm{NaCl} 0,9$ p. 100 (6 jours), glucose 10 p. 100 (6 jours) et $\mathrm{NaCl} 0,9$ p. 100 (4 jours).

\section{Collecte de suc pancréatique et détermination des activités enzymatiques.}

Les modalités de récolte du suc pancréatique ont été décrites précédemment (Simoes Nunes et Corring, 1979). Dans les essais de perfusion intraveineuse chez l'animal à jeun, six échantillons de $10 \mathrm{ml}$ chacun ont été prélevés (un par heure). Chez l'animal nourri, quatre échantillons de $10 \mathrm{ml}$ ont été prélevés respectivement à $9 \mathrm{~h}$, $9 \mathrm{~h} 30,11 \mathrm{~h}$ et $15 \mathrm{~h}$ de la journée. Le volume de suc pancréatique écoulé a été systématiquement mesuré.

Dans chacun des échantillons de suc pancréatique, il a été procédé aux déterminations des protéines totales et des activités enzymatiques de la chymotrypsine, de l'amylase et de la lipase selon les méthodes ciłées précédemment (Simoes Nunes et Corring, 1979). Les activités enzymatiques sont exprimées en activités totales (activité enzymatique $x$ volume de suc) et en activités spécifiques (activité enzymatique/mg de protéines totales). D'autre part, dans les essais de perfusion intraveineuse chez l'animal nourri, il a été procédé à la détermination de la glycémie (Huggett et Nixon, 1957) sur des échantillons de sang prélevés respectivement $15 \mathrm{~min}$ avant le repas de $9 \mathrm{~h} 00$ et 30 , 120 et $360 \mathrm{~min}$ après celui-ci.

Le suc pancréatique était réintroduit en permanence dans la lumière intestinale après échantillonnage, éliminant ainsi la possibilité de stimulation de cette sécrétion causée par l'absence d'enzymes pancréatiques et en particulier des protéases dans la lumière intestinale (Green et Lyman, 1972 ; Corring, 1974 ; Ihse, Lilja et Lundquist, 1977).

Toutes les données ont été interprétées statistiquement (tesł « $t$ »de Student).

\section{Résultats.}

1) Perfusions intraveineuses de $\mathrm{NaCl} 0,9$ p. 100 ef de glucose 5 et 15 p. 100 pratiquées chez l'animal à jeun. - Etant donné les importantes variations individuelles (supérieures à 50 p. 100), les valeurs obtenues pendant la première heure de perfusion intraveineuse ( $\mathrm{NaCl} \mathrm{0,9}$ p. 100) ont été retenues comme base 100 et les valeurs correspondantes aux $5 \mathrm{~h}$ suivantes sont exprimées en pourcentage de la valeur basale.

Les résultats sont présentés dans les tableaux 1 et 2 . La perfusion intraveineuse de glucose 5 et 15 p. 100 entraîne une diminution significative du volume de suc pancréa- 
TABLEAU 1

Volume $(\mathrm{ml})$, proféines fofoles $(\mathrm{mg})$ ef activités enzymatiques tofales (activité enzymatique $\times$ volume) du suc pancréatique recueilli pendant $6 \mathrm{~h}$ de perfusion intraveineuse de solutions de chlorure de sodium 0,9 p. 100 ef de glucose 5 p. $100(8,33 \mathrm{ml} / \mathrm{min})$

\begin{tabular}{|c|c|c|c|c|c|}
\hline & Volume & $\begin{array}{l}\text { Protéines } \\
\text { totales }\end{array}$ & $\begin{array}{l}\text { Chymotryp- } \\
\text { sine }\end{array}$ & Amylase & Lipase \\
\hline $\begin{array}{c}\mathrm{NaCl} 0,9 \\
(1 \text { h) } \\
\left(^{(}\right)\end{array}$ & $79,3 \pm 15$ & $216 \pm 54$ & $5306 \pm 1312$ & $788512 \pm 230609$ & $8172 \pm 1356$ \\
\hline $\begin{array}{l}\text { Glucose } 5 \text { p. } 100 \ldots \\
\left(1 \text { h) }\left(^{2}\right)\right. \\
\text { NaCl } 0,9 \text { p. } 100 \ldots \\
\left(1 \text { h) }\left(^{2}\right)\right. \\
\text { Glucose } 5 \text { p. } 100 \ldots \\
\left(1 \text { h) }\left(^{2}\right)\right. \\
\text { Glucose } 5 \text { p. } 100 \ldots \\
\left(1 \text { h) }\left(^{2}\right)\right. \\
\text { NaCl } 0,9 \text { p. } 100 \ldots \\
\left(1 \text { h) }\left(^{2}\right)\right.\end{array}$ & $\begin{array}{c}56 \pm 5 \\
P<0,001 \\
96 \pm 6 \\
N S \\
40 \pm 4 \\
P<0,001 \\
45 \pm 4 \\
P<0,001 \\
109 \pm 3 \\
P<0,025\end{array}$ & $\begin{array}{c}84 \pm 12 \\
N S \\
104 \pm 11 \\
N S \\
56 \pm 15 \\
P<0,05 \\
54 \pm 6 \\
P<0,001 \\
117 \pm 4 \\
P<0,005\end{array}$ & $\begin{array}{c}90 \pm 15 \\
\text { NS } \\
106 \pm 11 \\
\text { NS } \\
65 \pm 20 \\
\text { NS } \\
62 \pm 9 \\
P<0,001 \\
122 \pm 6 \\
P<0,0025\end{array}$ & $\begin{array}{c}65 \pm 8 \\
P<0,01 \\
99 \pm 11 \\
N S \\
42 \pm 12 \\
P<0,005 \\
46 \pm 5 \\
P<0,001 \\
115 \pm 6 \\
N S\end{array}$ & $\begin{array}{c}78 \pm 10 \\
\text { NS } \\
103 \pm 10 \\
N S \\
57 \pm 15 \\
P<0,05 \\
53 \pm 5 \\
P<0,001 \\
112 \pm 3 \\
P<0,01\end{array}$ \\
\hline
\end{tabular}

(1) Les valeurs obtenues pendant la première heure de perfusion $(\mathrm{NaCl})$ ont été retenues comme base 100 et les valeurs correspondantes aux 5 h suivantes $\left({ }^{2}\right)$ sont exprimées en pourcentage de la valeur basale.

\section{TABLEAU 2}

Volume $(\mathrm{ml})$, protéines tołales $(\mathrm{mg})$ et activités enzymatiques totales (activité enzymatique $\times$ volume) du suc pancréatique recueilli pendant $6 \mathrm{~h}$ de perfusion intraveineuse de solutions de chlorure de sodium 0,9 p. 100 ef de glucose 15 p. $100(8,33 \mathrm{ml} / \mathrm{min})$

\begin{tabular}{|c|c|c|c|c|c|}
\hline & Volume & $\begin{array}{l}\text { Protéines } \\
\text { totales }\end{array}$ & Chymotrypsine & Amylase & Lipase \\
\hline $\begin{array}{c}\mathrm{NaCl} 0,9 \text { p. } 100 \\
(1 \mathrm{~h})\left({ }^{1}\right)\end{array}$ & $150 \pm 48$ & $578 \pm 121$ & $13130 \pm 3022$ & $1207007 \pm 280847$ & $24995 \pm 5919$ \\
\hline $\begin{array}{l}\text { Glucose } 15 \text { p. } 100 . \\
(1 \mathrm{~h})\left({ }^{2}\right) \\
\text { NaCl } 0,9 \text { p. } 100 \\
\left(1 \text { h) }\left({ }^{2}\right)\right. \\
\text { Glucose } 15 \text { p. } 100 . \\
\left(1 \text { h) }\left(^{2}\right)\right. \\
\text { Glucose } 15 \text { p. } 100 . \\
\left(1 \text { h) }\left(^{2}\right)\right. \\
\text { NaCl } 0,9 \text { p. } 100 \ldots \\
\left(1 \text { h) }\left(^{2}\right)\right.\end{array}$ & $\begin{array}{r}40 \pm 2 \\
P<0,001 \\
99 \pm 5 \\
N S \\
44 \pm 10 \\
P<0,005 \\
40 \pm 7 \\
P<0,001 \\
110 \pm 4 \\
P<0,05\end{array}$ & $\begin{array}{c}29 \pm 7 \\
P<0,001 \\
99 \pm 10 \\
N S \\
46 \pm 11 \\
P<0,005 \\
40 \pm 10 \\
P<0,005 \\
90 \pm 2 \\
P<0,001\end{array}$ & $\begin{array}{c}33 \pm 8 \\
P<0,001 \\
102 \pm 12 \\
N S \\
53 \pm 14 \\
P<0,025 \\
49 \pm 14 \\
P<0,025 \\
90 \pm 3,5 \\
P<0,005\end{array}$ & $\begin{array}{r}31 \pm 10 \\
P<0,001 \\
99 \pm 11 \\
N S \\
40 \pm 10 \\
P<0,001 \\
44 \pm 9 \\
P<0,001 \\
89 \pm 2 \\
P<0,005\end{array}$ & $\begin{array}{c}29 \pm 6 \\
P<0,001 \\
101 \pm 9 \\
N S \\
45 \pm 12 \\
P<0,005 \\
40 \pm 10 \\
P<0,005 \\
88 \pm 1 \\
P<0,001\end{array}$ \\
\hline
\end{tabular}

(') et ( $\left.{ }^{2}\right)$ cf. légende tableau 1.

tique excrété (-53 p. 100 en moyenne avec le glucose 5 p. 100 et -58 p. 100 en moyenne avec le glucose 15 p. 100), des protéines totales excrétées (-35 p. 100 en moyenne avec le glucose 5 p. 100 et -61 p. 100 en moyenne avec le glucose 15 p. 100), de la chymotrypsine totale excrétée ( -28 p. 100 en moyenne avec le glucose 5 p. 100 et -55 p. 100 en moyenne avec le glucose 15 p. 100), de l'amylase totale excrétée ( -49 p. 100 en moyenne avec le glucose 5 p. 100 et -65 p. 100 en moyenne avec le 
glucose 15 p. 100) et de la lipase totale excrétée (-38 p. 100 en moyenne arec le glucose 5 p. 100 et -62 p. 100 en moyenne avec le glucose 15 p. 100). En ce qui concerne les activités spécifiques des trois enzymes (łabl. 3 et 4), celle de l'amylase diminue signi-

\section{TABLEAU 3}

Activités enzymatiques spécifiques (/mg de protéines) du suc pancréatique recueilli pendant $6 \mathrm{~h}$ de perfusion intraveineuse de solutions de chlorure de sodium 0,9 p. 100 ef de glucose 5 p. $100(8,33 \mathrm{ml} / \mathrm{min})$

\begin{tabular}{|c|c|c|c|}
\hline & Chymotrypsine & Amylase & Lipase \\
\hline$\underset{(1 \text { h })\left({ }^{(1)}\right.}{\mathrm{NaCl} 0,9 \text { p. } 100 \ldots \ldots \ldots}$ & $25 \pm 1$ & $3615 \pm 647$ & $42 \pm 7$ \\
\hline $\begin{array}{l}\text { Glucose } 5 \text { p. } 100 \ldots \ldots \ldots \\
\left(1 \text { h) }\left({ }^{2}\right)\right. \\
\text { NaCi } 0,9 \text { p. } 100 \ldots \ldots \ldots \\
\left(1 \text { h) }\left({ }^{2}\right)\right. \\
\text { Glucose } 5 \text { p. } 100 \ldots \ldots \ldots \\
\left(1 \text { h) }\left({ }^{2}\right)\right. \\
\text { Glucose } 5 \text { p. } 100 \ldots \ldots \ldots \ldots \\
\left(1 \text { h) }\left(^{2}\right)\right. \\
\text { NaCi } 0,9 \text { p. } 100 \ldots \ldots \ldots \\
\left(1 \text { h) }\left({ }^{2}\right)\right.\end{array}$ & $\begin{array}{l}106 \pm 2 \\
P<0,05 \\
101 \pm 2 \\
N S \\
113 \pm 3 \\
P<0,01 \\
112 \pm 5 \\
N S \\
103 \pm 2 \\
N S\end{array}$ & $\begin{array}{l}79 \pm 4 \\
P<0,005 \\
94 \pm 22 \\
P<0,005 \\
75 \pm 6 \\
P<0,005 \\
86 \pm 3 \\
P<0,005 \\
97 \pm 3 \\
\text { NS } 3\end{array}$ & $\begin{array}{l}94 \pm 3 \\
N S \\
98 \pm 2 \\
N^{ \pm S} \\
99 \pm 4 \\
N S \\
98 \pm 5 \\
N^{ \pm S} \\
95 \pm 3 \\
N S\end{array}$ \\
\hline
\end{tabular}

(1) et ${ }^{(2)}$ cf. légende tableau 1.

TABLEAU 4

Activités enzymatiques spécifiques (/mg protéines) du suc pancréatique recueilli pendant $6 \mathrm{~h}$ de perfusion intraveineuse de solutions de chlorure de sodium 0,9 p. 100 ef de glucose 15 p. $100(8,33 \mathrm{ml} / \mathrm{min})$

\begin{tabular}{|c|c|c|c|}
\hline & Chymotrypsine & Amylase & Lipase \\
\hline $\begin{array}{l}\mathrm{NaCl} 0,9 \text { p. } 100 \ldots \ldots \\
(1 \mathrm{~h})\left({ }^{(1)}\right.\end{array}$ & $22 \pm 1$ & $2191 \pm 460$ & $43 \pm 7$ \\
\hline $\begin{array}{l}\text { Glucose } 15 \text { p. } 100 \ldots \ldots \\
\left(1 \text { h) }\left(^{2}\right)\right. \\
\text { NaCl } 0,9 \text { p. } 100 \ldots \ldots \\
\left(1 \text { h) }\left(^{2}\right)\right. \\
\text { Glucose } 15 \text { p. } 100 \ldots \ldots \\
\left(1 \text { h) }\left(^{2}\right)\right. \\
\text { Glucose } 15 \text { p. } 100 \ldots \ldots \\
\left(1 \text { h) }\left(^{2}\right)\right. \\
\text { NaCl } 0,9 \text { p. } 100 \ldots \ldots \\
\left(1 \text { h) }\left(^{2}\right)\right.\end{array}$ & $\begin{array}{l}113 \pm 3 \\
P<0,01 \\
102 \pm 1 \\
N S \\
114 \pm 3 \\
P<0,01 \\
11.5 \pm 8 \\
N S \\
99 \pm 4 \\
N S\end{array}$ & $\begin{array}{c}82 \pm 1 \\
\mathrm{P}<0,001 \\
100 \pm 1 \\
\mathrm{NS} \\
86 \pm 3 \\
\mathrm{P}<0,005 \\
84 \pm 2 \\
\mathrm{P}<0,001 \\
98 \pm 4 \\
\mathrm{NS}\end{array}$ & $\begin{array}{c}101 \pm 2 \\
N S \\
102 \pm 2 \\
N S \\
98 \pm 2 \\
N S \\
99 \pm 2 \\
N S \\
97 \pm 2 \\
N S\end{array}$ \\
\hline
\end{tabular}

(1) et $\left({ }^{2}\right)$ cf. légende tableau 1.

ficativement ( -20 p. 100 en moyenne avec le glucose 5 p. 100 et -16 p. 100 en moyenne avec le glucose 15 p. 100), celle de la chymotrypsine augmente $(+10$ p. 100 en moyenne arec le glucose 5 p. 100 et +14 p. 100 en moyenne avec le glucose 15 p. 100), tandis que l'activité spécifique de la lipase n'est pas affectée par les deux types de perfusion intraveineuse. 
2) Perfusions intraveineuses de $\mathrm{NaCl} 0,9$ p. 100 ef de glucose 10 p. 100 pratiquées chez l'anima! nourri. - Les effets de la perfusion intraveineuse prolongée de glucose sur le

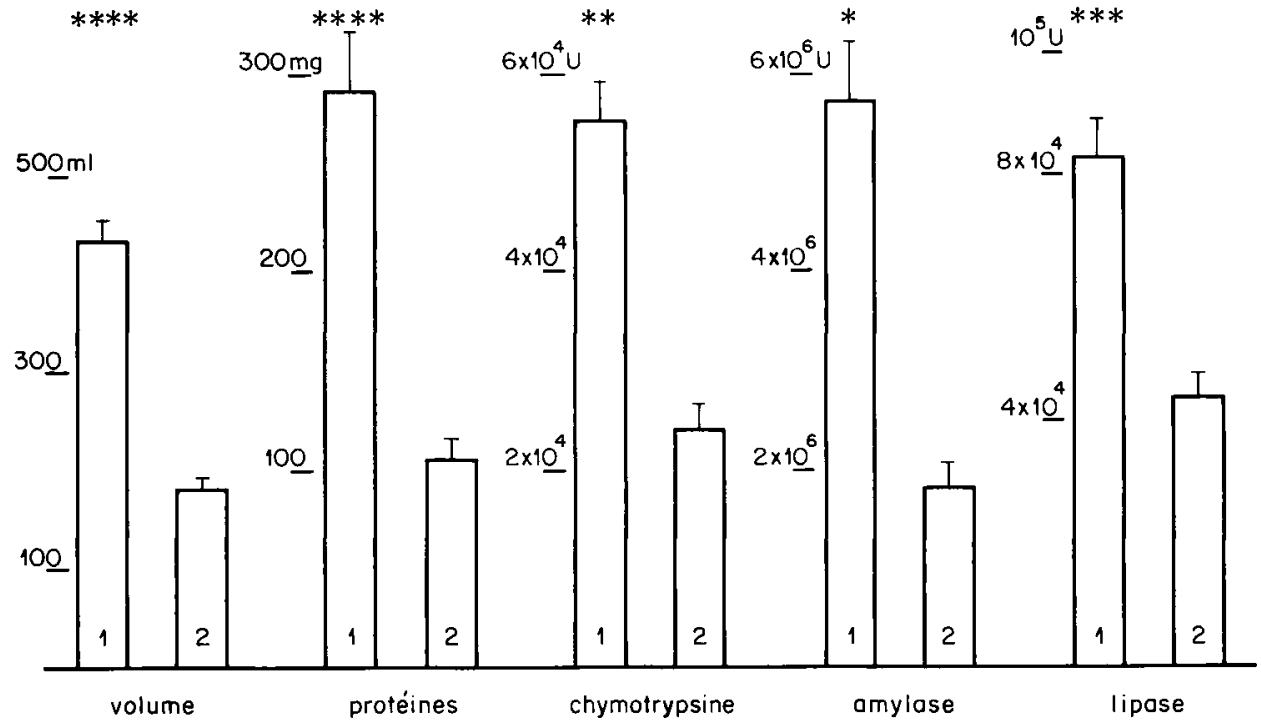

FIG. 1. - Effets sur la sécrétion pancréatique exocrine (volume, protéines totales et activités enzymatiques totales) de la perfusion intraveineuse d'une solution de glucose associée à la consommation du repas. 1. Période de perfusion de $\mathrm{NaCl} 0,9$ p. $100(8,33 \mathrm{ml} / \mathrm{min}$ sur $6 \mathrm{~h}$ pendant $6+4$ iours $) ; 2$. Période de perfusion de glucose 10 p. 100 (id. pendant 6 jours).

* $\mathrm{P}<0,01$; ** $\mathrm{P}<0,005$; *** $\mathrm{P}<0,0025 ;{ }^{* * * *} \mathrm{P}<0,001$.

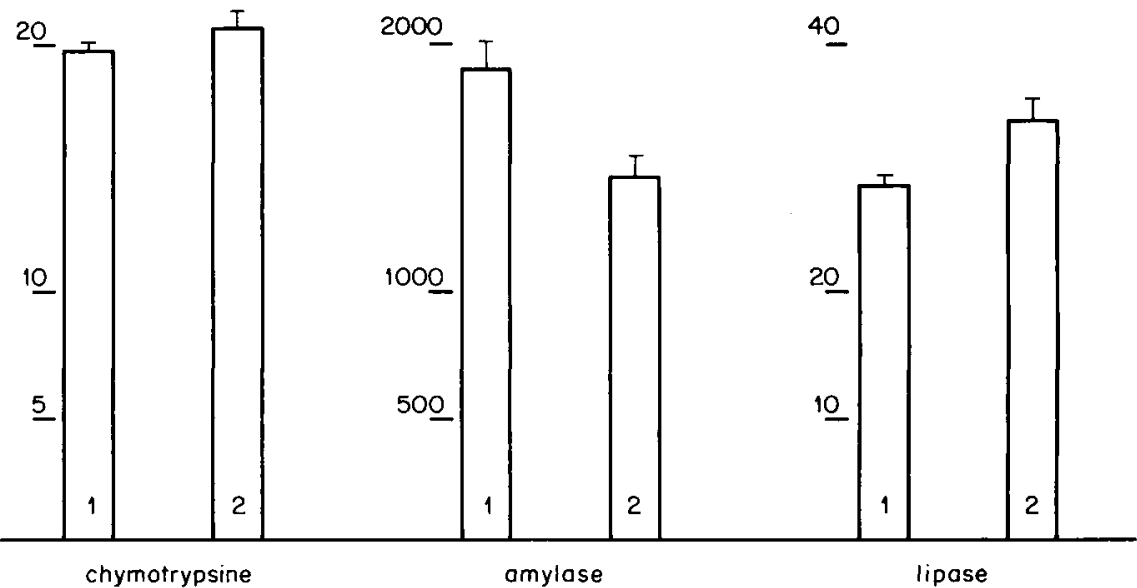

FIG. 2. - Effels sur les activités enzymatiques spécifiques (/mg protéines) de la perfusion infraveineuse d'une solution de glucose associée d̀ la consommation du repas.

1 et $2:$ (cf. légende fig. 1).

$* P<0,05$. 
volume, les protéines totales, la chymotrypsine, l'amylase ef la lipase totales excrétés sont représentés dans la figure 1. Chacune des valeurs rapportées est la moyenne des données obtenues pendant les périodes de 10 jours de perfusion de $\mathrm{NaCl} \mathrm{0,9}$ p. 100 et de 6 jours de perfusion de glucose 10 p. 100. La perfusion intraveineuse de glucose associée à la consommation d'un repas se traduit par rapport à la perfusion intraveineuse de $\mathrm{NaCl}$ 0,9 p. 100 par une diminution en moyenne de 58 p. 100 du volume de suc pancréatique sécrété, de 64 p. 100 des protéines totales excrétées, de 56 p. 100 de l'activité totale de la chymotrypsine totale excrétée, de 67 p. 100 de celle de l'amylase et de 48 p. 100 de celle de la lipase. En ce qui concerne les activités spécifiques des trois enzymes (fig. 2), celle de la chymotrypsine augmente non significativement de 4 p. 100 , celle de l'amylase diminue significativement de 22 p. 100 et celle de la lipase augmente significativement de 25 p. 100 sous l'effet de la perfusion intraveineuse de glucose associée à la consommation d'un repas.

La glycémie moyenne pendant les six heures de perfusion intraveineuse et sur toute la durée de l'étude (tabl. 5) a été de $932,1 \pm 33,1 \mathrm{mg} / \mathrm{l}$ sous glucose et de

\section{TABLEAU 5}

Glycémie chez l'animal nourri et recevant des perfusions intraveineuses de solutions de $\mathrm{NaCl} 0,9$ p. 100 et de glucose 10 p. $100(8,33 \mathrm{ml} / \mathrm{min}$ pendant $6 \mathrm{~h})$

\begin{tabular}{ccc}
\hline \multicolumn{3}{c}{ Glucose $(\mathrm{mg} / \mathrm{l})$} \\
\hline Basale $\left(^{(1)}\right.$ & $\mathrm{NaCl} 0,9 \mathrm{p} .100\left(^{2}\right)$ & Glucose $10 \mathrm{p.} 100\left(^{3}\right)$ \\
$849,2 \pm 9,5 a$ & $897,2 \pm 13,2^{b}$ & $932,1 \pm 33,1 b$ \\
& $\mathrm{P}<0,005$ & $\mathrm{P}<0,05$ \\
\hline
\end{tabular}

(1) Moyenne \pm écart-type des valeurs correspondantes à $15 \mathrm{~min}$ avant le début du repas ef des perfusions. ( $\left.{ }^{2}\right)$ Moyenne \pm écart-type des valeurs correspondantes aux $6 \mathrm{~h}$ de perfusion de $\mathrm{NaCl}$ 0,9 p. 100 (pendant $6+4$ jours). $\left({ }^{3}\right)$ Moyenne \pm écart-type des valeurs correspondantes aux $6 \mathrm{~h}$ de perfusion de glucose 10 p. 100 (pendant 6 jours). $a, b$ : Les valeurs portant la même lettre ne sont pas significativement différentes.

$897,2 \pm 13,2 \mathrm{mg} / /$ sous $\mathrm{NaCl}$ 0,9 p. 100 (différence non significative). Les deux teneurs sanguines en glucose sont statistiquement différentes de la glycémie basale $(849,2 \pm 9,5 \mathrm{mg} / \mathrm{l})$. Il faut souligner que dans les deux cas, il s'agit de glycémie postprandiale.

\section{Discussion.}

L'ensemble des données présentées montrent que le glucose administré par voie intraveineuse de façon prolongée a des effets semblables à ceux décrits précédemment (Simoes Nunes et Corring, 1980) après injection unique. Outre la diminution du volume et des protéines totales excrétées, on constate que les activités totales des enzymes sont inhibées. L'effet inhibiteur de l'administration parentérale de glucose sur la sécrétion pancréatique exocrine confirme chez le porc, les observations déjà anciennes de Brown Scott ef al. (1941) et celles plus récentes de Nakajima et Magee (1970), de Towne, Hamilton et Stephenson (1973) et de Saito et al. (1978), chez le chien, de Call, Mitchell et Little (1975) chez le mouton et de Dudrick ef al. (1970) chez l'homme. 
Par ailleurs, Hughes, Bates et Dowling (1978) onf récemment montré que chez le chien alimenté par voie parentérale avec ou sans administration quotidienne de cholécystokinine-pancréozymine (CCK-PZ) ef de sécrétine, la capacité de réponse du pancréas exocrine à une stimulation par ces deux hormones était maintenue en ce qui concerne le volume, les protéines totales et les bicarbonates excrétés, mais par contre l'excrétion de l'amylase était très significativement diminuée. Brever et al. (1979) ont montré aussi que chez le rat ce type d'alimentation diminue très fortement la quantité d'amylase et de trypsine exportée par la glande, cela étant accompagné d'une augmentation de la concentration enzymatique du tissu pancréatique.

Dans la présente éfude, l'amylase apparaît comme l'enzyme la plus sensible à l'action du glucose administré par voie intraveineuse parce qu'aussi bien chez l'animal à jeun que nourri, ses activités totale et spécifique sont les plus affectées. Un résultat semblable a été trouvé par Nakajima et Magee (1970) chez le chien.

La pression osmotique des solutions de glucose 10 p. $100(555,6 \mathrm{~m} \mathrm{Osm/I)}$ et $15 \mathrm{p}$. $100(833,4 \mathrm{~m}$ Osm $/ 1)$ plus élevée que celle du plasma sanguin, ne peut pas être responsable des effets produits, dans la mesure où l'inhibition de la sécrétion pancréatique exocrine a été observée également au cours de la perfusion de la solution isotonique de glucose. De même Saito ef al. (1978) ont montré que la pression osmotique des solutions employées chez le chien (glucose 20 et 30 p. 100) n'était pas responsable des effets inhibiteurs observés.

La bibliographie sur les relations entre la glycémie et la sécrétion pancréatique exocrine est très souvent contradictoire. Dans cette étude, la glycémie n'a été déterminée que pendant les perfusions intraveineuses chez l'animal recevant deux repas par jour et les résultats obtenus montrent que la perfusion de glucose n'entraîne qu'une augmentation modérée et non significative $(34,9 \mathrm{mg} / \mathrm{l})$ de la glycémie post-prandiale par rapport à celle, post-prandiale également, observée avec la perfusion de la solution de chlorure de sodium 0,9 p. 100. Chez le rat rendu diabétique par l'alloxane, il y a effondrement de l'activité amylasique du tissu pancréatique (Palla, Ben Abdeljlil et Desnuelle, 1968 ; Adler et Kern, 1975). Cette chute de l'activité amylolytique du pancréas est accompagnée d'une augmentation de l'activité du chymotrypsinogène et de la lipase pancréatiques ; le traitement par l'insuline étant capable de restaurer l'activité de l'amylase et augmente aussi celle du chymotrypsinogène ef de la lipase (Palla, Ben Abdeljlil et Desnuelle, 1968).

Crider et al. (1956) ont rapporté une augmentation de la concentration en amylase dans le suc pancréatique du chien à la suite d'une injection intraveineuse de $20 \mathrm{ml}$ de glucose en solution à 50 p. 100 . Deux remarques peuvent toutefois être faites : d'une part, leur étude n'a comporté que 3 essais ef parallèlement il y a eu diminution du volume de suc excrété après l'injection. Ainsi, si l'on calcule les données de Crider et al. (1956) en termes d'activité totale d'amylase, elle a été, après l'injection intraveineuse de glucose, en moyenne 12,35 p. 100 inférieure à celle observée pendant la période basale correspondante. Les résultats de Crider et al. (1956) ne seraient donc qu'apparemment contradictoires.

Chez le rat, Lavau, Bazin et Herzog (1974) ont observé une augmentation de l'activité spécifique de l'amylase et une diminution de l'activité spécifique de la lipase dans le tissu pancréatique après perfusion intraveineuse de glucose 30 p. 100 pendant 5 jours, reproduisant ainsi l'adaptation de la sécrétion pancréatique exocrine observée avec 
une quantité importante d'amidon ou de glucose dans le régime alimentaire. Adler et Kern (1975), après une perfusion de glucose 50 p. 100 observent aussi une augmentation de l'activité spécifique de l'amylase. La contradiction qui existe entre ces résultats et ceux rapportés dans ce travail chez le porc peut, à notre sens, s'expliquer en grande partie par les quantités de glucose administrées aux animaux. En effet, chez le rat Lavau, Bazin ef Herzog (1974) ef Adler et Kern (1975) ont injecté des quantifés de glucose nettement supérieures à celles administrées chez le porc. Ces dernières se rapprochent certainement beaucoup plus de l'apport nutritif glucidique observé chez l'animal adapté à un régime riche en amidon. Il est fort peu probable que les résultats contradictoires obtenus chez le rat et le porc proviennent du matériel organique étudié : tissu pancréatique chez le rat et suc pancréatique chez le porc. En effet, les changements de régime induisent des modifications adaptatives aussi bien au niveau du tissu que dans le suc excrété (Ben Abdeljlil, Visani et Desnuelle, 1963).

En conclusion, les résultats rapportés dans la présente étude et ceux de Nakajima et Magee (1970) montrent que le glucose administré par voie intraveineuse de façon prolongée n'est pas capable de reproduire les modifications de type adaptatif de l'amylase pancréatique qu'il provoque quand il est administré en quantités équivalentes par voie orale ou duodénale (Howard et Yudkin, 1963 ; Deschodt-Lanckman et al., 1971 ; Simoes Nunes et Corring, 1979).

Reçu en janvier 1981. Accepté en avril 1981.

\section{Références}

ADLER G., KERN H. F., 1975. Regulation of exocrine pancreafic secretory process by insulin in vivo. Horm. Metab. Res., 7, 290-296.

BEHRMAN H., KARE M., 1969. Adaptation of canine pancreatic enzymes to diet composition. J. Physiol., 205, 667-676.

BEN ABDELJLIL A., VISANI A. M., DESNUELLE P., 1963. Adaptation of the exocrine secretion of rat pancreas to the composition of the diet. Bioch. biophys. Res. Commun., 10, 112-116.

BREUER R. S., HATOFF D. E., HUGHES C. A., DOWLING R. H., 1979. Is CCK trophic to small bowel and/or pancreas ? : a study in rats during exclusive parenteral nutrition (EPN). Gut, 20, A 911.

BROWN SCOTT V., COLLIGNON U. J., BUGEL H. J., JOHNSON G. C., 1941. The relation of external pancreatic secretion to variations in blood sugar. Am. J. Physiol., 134, 208-218.

CALL J. L., MITCHELL Jr G. E., LITTLE C. O., 1975. Response of ovine pancreatic amylase to elevated blood glucose. J. anim. Sci., 41, 1717-1721.

CLARY J. J., MITCHELL G. E., LITTLE C. O., BRADLEY N. W., 1969. Pancreatic amylase activity from ruminants fed different rations. Can. J. Physiol. Pharmacol., 47, 161-164.

CORRING T., 1974. Régulation de la sécrétion pancréatique par rétro-inhibition négative chez le porc. Ann. Biol. anim. Bioch. Biophys., 14, 487-498.

CORRING T., 1975. Adaptation de la sécrétion du pancréas exocrine au régime alimentaire chez le porc. Physiologie comparée, étude expérimentale et mécanismes. Th. Etat, Univ. Paris VI.

CORRING T., 1977. Possible role of hydrolysis products of the dietary components in the mechanism of the exocrine pancreatic adaptation to the diet. WId. Rev. Nutr. Diet., 27, 132-144.

CORRING T., AUMAITRE A., RÉRAT A., 1972. Fistulation permanente du pancréas exocrine chez le porc. Application : réponse de la sécrétion pancréatique au repas. Ann. Biol. anim. Bioch. Biophys., 12, 109-124.

CRIDER J. O., CONLY S. S., DORCHESTER J. E. C., THOMAS J. E., 1956. Effect of intravenous injection of hypertonic glucose solution on external secretion of the pancreas. Am. J. Physiol., 186, 187-189. 
DESCHODT-LANCKMAN M., ROBBERECHT P., CAMUS J., CHRISTOPHE J., 1971, Shorf-term adaptation of pancreatic hydrolases to nutritional and physiological stimuli in adult rats. Biochimie, 53, 789-796.

DUDRICK S. J., WILMORE D. W., STEIGER E., MACKIE J. A., FITTS W. J., $1970 . \quad$ Spontaneous closure of traumatic pancreato-duodenal fistulas with total intravenous nutrition. J. Trauma., 10, 542543.

FELBER J. P., ZERMATTEN A., DICK J., 1974. Modulation by food, of hormonal system regulating rat pancreatic secretion. Lancet, 2, 185-187.

GREEN G. M., LYMAN R. L., 1972. Feedback regulation of pancreatic enzyme secretion as a mechanism for trypsin-inhibitor-induced hypersecretion in rats. Proc. Soc. exp. Biol. Med., 140, 6-12.

GROSSMAN M. I., GREENGARD H., IVY A. C., 1943. The effects of diefary composition on pancreatic enzymes. Am. J. Physiol., 138, 676-682.

HOWARD F., YUDKIN J., 1963. Effect of dietary change upon the amylase and trypsin activities of the rat pancreas. Br. J. Nutr., 17, 281-294.

HUGGETT A. S. G., NIXON D. A., 1957. Enzymatic determination of blood glucose. Bioch. J., 66, 12 pp.

HUGHES C. A., BATES T., DOWLING R. H., 1978. Cholecystokinin and secretin prevent the intestinal mucosal hypoplasia of total parenteral nutrition in the dog. Gastroenferology, 75, 34-41.

IMONDI A. R., BIRD F. H., 1967. Effects of dietary protein level on growth and proteolytic activity of the avian pancreas. J. Nutr., 91, 421-428.

IHSE I., LILJA P., LUNDQUIST I., 1977. Feedback regulation of pancreatic enzyme secretion by intestinal trypsin in man. Digestion, 15, 303-308.

JOHNSON A., HURWITZ R., KRETCHMER N., 1977. Adaptation of rat pancreatic amylase and chymotrypsinogen to changes in diet. J. Nutr., 107, 87-96.

LAVAU M., BAZIN R., HERZOG J., 1974. Comparative effects of oral and parenteral feeding on pancreatic enzymes in the rat. J. Nutr., 104, 1432-1437.

NAKAJIMA S., MAGEE D. F., 1970. Inhibition of exocrine pancreatic secretion by glucagon and Dglucose given intravenously. Can. J. Physiol. Pharmacol., 48, 299-305.

PALLA J. C., BEN ABDELJLIL A., DESNUELLE P., 1968. Action de l'insuline sur la biosynthèse de l'amylase et de quelques autres enzymes du pancréas de rat. Biochim. biophys. Acta, 158, 25-35.

ROBBERECHT P., DESCHODT-LANCKMAN M., CAMUS J., BRUYLANDS J., CHRISTOPHE J., 1971. Rat pancreatic hydrolases from birth to weaning and dietary adaptation after weaning. Am. J. Physiol., 221, 376-381.

SAITO Y., TOKUTAKE K., MATSUNO S., NOTO N., HONDA T., SATO T., 1978. Effects of hypertonic glucose and amino acid infusions on pancreatic exocrine function. Tohuku J. exp. Med., 124, 99-115.

SIMOES-NUNES C., CORRING T., 1979. Pancreatic exocrine secretion in the pig following test meals of different composition and intra-duodenal loads of glucose and maltose. Horm. Metab. Res., 11, 346-351.

SIMOES-NUNES C., CORRING T., 1980. Rôle de la muqueuse duodénale dans l'adaption de l' $\alpha$-amylase pancréatique au régime alimentaire chez le porc. Reprod. Nutr. Dévelop., 20, 1237-1245.

TOWNE J. B., HAMILTON R. F., STEPHENSON Jr D. V., 1973. Mechanism of hyperalimentation in the suppression of upper gastrointestinal secretions. Am. J. Surg., 126, 714-716. 